Article

\title{
Existence of a Unique Fixed Point for Nonlinear Contractive Mappings
}

\author{
Simeon Reich * and Alexander J. Zaslavski \\ Department of Mathematics, The Technion-Israel Institute of Technology, Haifa 32000, Israel; \\ ajzasl@technion.ac.il \\ * Correspondence: sreich@technion.ac.il
}

Received: 6 December 2019; Accepted: 26 December 2019; Published: 1 January 2020

\begin{abstract}
In a recent work, we established the existence of a unique fixed point for nonlinear contractive self-mappings of a bounded and closed set in a Banach space. In the present paper we extend this result to the case of unbounded sets.
\end{abstract}

Keywords: Banach space; complete metric space; contractive mapping; fixed point; iterate

MSC: 47H09; 47H10; 54E35; 54E50

\section{Introduction}

For almost six decades, there has been considerable research activity regarding the fixed point theory of nonexpansive (that is, 1-Lipschitz) mappings. See, for instance, [1-15] and the references cited therein. This activity stems from Banach's classical result [16] concerning the existence of a unique fixed point for a strict contraction. In addition, it also concerns, inter alia, the convergence of (inexact) iterates of a nonexpansive mapping to one of its fixed points. Since that seminal result, many developments have taken place in this field including, in particular, studies of feasibility, common fixed point problems, and variational inequalities, which find important applications in engineering, medicine, and the natural sciences [14,15,17-21].

In a recent work of ours [22], we established the existence of a unique fixed point for nonlinear contractive self-mappings of a bounded and closed subset of a Banach space. In the present paper, we extend this result to the case of unbounded sets.

More precisely, in [22,23] we considered the following class of nonlinear mappings.

Assume that $(X,\|\cdot\|)$ is a Banach space and that $K \subset X$ is a bounded, closed, and convex set. Assume further, that $f: X \rightarrow[0, \infty)$ is a continuous function for which $f(0)=0$, the set $f(K-K)$ is bounded, and the following three properties are valid:

(i) For every positive number $\epsilon$, there is a positive number $\delta$ such that for each pair of points $x, y \in K$ satisfying $f(x-y) \leq \delta$, we have $\|x-y\| \leq \epsilon$;

(ii) For every number $\lambda \in(0,1)$, there exists a number $\phi(\lambda) \in(0,1)$ for which

$$
f(\lambda(x-y)) \leq \phi(\lambda) f(x-y) \text { for all } x, y \in K ;
$$

(iii) The function $(x, y) \mapsto f(x-y), x, y \in K$, is uniformly continuous on the set $K \times K$.

Denote by $\mathcal{A}$ the set of all continuous mappings $A: K \rightarrow K$ which satisfy

$$
f(A x-A y) \leq f(x-y) \text { for all } x, y \in K \text {. }
$$


For every pair of mappings $A, B \in \mathcal{A}$, define

$$
d(A, B):=\sup \{\|A x-B x\|: x \in K\} .
$$

Evidently, $(\mathcal{A}, d)$ is a complete metric space.

In [23] we established the existence of an everywhere dense and $G_{\delta}$ subset of $\mathcal{A}$, such that each one of its elements possesses a unique fixed point and all the iterates of such an element converge uniformly to this fixed point.

We remark in passing that the main result of [24] is a special case of this result of [23] for the case where $f=\|\cdot\|$. Clearly, the mappings considered in our papers are generalized nonexpansive mappings with respect to the function $f$. This approach, where the norm is replaced with a general function, had already been used in $[25,26]$, in the study of generalized best approximation problems.

In [22] we improved the results of [23]. Namely, we introduced a notion of a contractive mapping, we showed that most of the mappings in $\mathcal{A}$ (in the sense of Baire category) are contractive, that every contractive mapping possesses a unique fixed point, and that all its iterates converge to this point uniformly. Note that all these results were obtained for a bounded set $K$. In the present paper we extend one of the main results of [22] to unbounded sets. More precisely, we show that even if $K$ is unbounded, every contractive self-mapping of $K$ possesses a unique fixed point and that all its iterates converge to this point, uniformly on bounded subsets of $K$. Moreover, for this result we do not need property (ii).

\section{Main Result}

Assume that $(X,\|\cdot\|)$ is a Banach space and that $K$ be a nonempty and closed subset of $X$. Assume further that $f: X \rightarrow[0, \infty)$ is a continuous function with $f(0)=0$ and that the following two properties are valid:

(P1) For every positive number $\epsilon$, there is a positive number $\delta$ such that for every pair of points $x, y \in K$ satisfying $f(x-y) \leq \delta$, we have $\|x-y\| \leq \epsilon$;

(P2) The function $(x, y) \mapsto f(x-y), x, y \in K$, is uniformly continuous on the set $K \times K$ and for each point $\xi \in K$, the function $f(x-\xi), x \in D$, is bounded on every bounded set $D \subset K$.

Assume that $A: K \rightarrow K$ is a continuous mapping, $\psi:[0, \infty) \rightarrow[0,1]$ is a decreasing function satisfying

$$
\psi(t)<1 \text { for every positive number } t
$$

and that

$$
f(A x-A y) \leq \psi(f(x-y)) f(x-y) \text { for each pair of points } x, y \in K .
$$

In other words, the mapping $A$ is contractive [13]. We denote the identity operator by $A^{0}$.

In Section 3 we establish the following result.

Theorem 1. The mapping $A$ has a unique fixed point $x_{A} \in K$ and $A^{i} x \rightarrow x_{A}$ as $i \rightarrow \infty$ for all $x \in K$, uniformly on bounded subsets of $K$.

Note that in [27] a particular case of this theorem was obtained for $f(x)=\|x\|$.

\section{Proof of Theorem 1}

Let $x \in K$. In view of (1), for every integer $n \geq 0$, we have

$$
\begin{aligned}
f\left(A^{n+1} x-A^{n+2} x\right) & \leq \psi\left(f\left(A^{n} x-A^{n+1} x\right)\right) f\left(A^{n} x-A^{n+1} x\right) \\
& \leq f\left(A^{n} x-A^{n+1} x\right) .
\end{aligned}
$$


We claim that

$$
\lim _{n \rightarrow \infty} f\left(A^{n} x-A^{n+1} x\right)=0 .
$$

Suppose to the contrary that this does not hold. Then by (2), there exists $\epsilon>0$ such that

$$
f\left(A^{n} x-A^{n+1} x\right) \geq \epsilon \text { for all integers } n \geq 0 .
$$

Since the function $\psi$ is decreasing, it follows from (2) and (3) that for every integer $n \geq 0$,

$$
f\left(A^{n+1} x-A^{n+2} x\right) \leq \psi(\epsilon) f\left(A^{n} x-A^{n+1} x\right) .
$$

This implies, in its turn, that $\lim _{n \rightarrow \infty} f\left(A^{n} x-A^{n+1} x\right)=0$. This equality contradicts relation (3). Therefore,

$$
\lim _{n \rightarrow \infty} f\left(A^{n} x-A^{n+1} x\right)=0 \text { for each } x \in K,
$$

as claimed.

Next, we show that the following property holds:

(P3) for every positive number $\epsilon$, there is a positive number $\delta$ such that for every pair of points $x, y \in K$ which satisfy

$$
f(x-A x) \leq \delta \text { and } f(y-A y) \leq \delta,
$$

we have

$$
\|x-y\| \leq \epsilon .
$$

Let $\epsilon>0$. By property (P1), there is

$$
\delta_{1} \in(0, \epsilon)
$$

for which

$$
\|x-y\| \leq \epsilon \text { for all } x, y \in K \text { satisfying } f(x-y) \leq \delta_{1} .
$$

Property (P2) implies that there exists a number

$$
\delta_{2} \in\left(0, \delta_{1}\right)
$$

such that

$$
\left|f\left(z_{1}-z_{2}\right)-f\left(\xi_{1}-\xi_{2}\right)\right| \leq\left(1-\psi\left(\delta_{1}\right)\right) \delta_{1} / 2
$$

for all $z_{1}, z_{2}, \xi_{1}, \xi_{2} \in K$ satisfying

$$
\left\|z_{i}-\xi_{i}\right\| \leq \delta_{2}, i=1,2 .
$$

By property (P1), there is

$$
\delta \in\left(0, \delta_{2}\right)
$$

such that the following property holds:

(P4) for each $z_{1}, z_{2} \in K$, if $f\left(z_{1}-z_{2}\right) \leq \delta$, then $\left\|z_{1}-z_{2}\right\| \leq \delta_{2}$.

Let $x, y \in K$ satisfy

$$
f(x-A x) \leq \delta \text { and } f(y-A y) \leq \delta .
$$

We will show that

$$
\|x-y\| \leq \epsilon .
$$

In view of (5), it is sufficient to prove that

$$
f(x-y) \leq \delta_{1} .
$$


Suppose, to the contrary, that this inequality does not hold. Then

$$
f(x-y)>\delta_{1} .
$$

Since the function $\psi$ is decreasing, relations (2) and (8) imply that

$$
f(A x-A y) \leq \psi(f(x-y)) f(x-y) \leq \psi\left(\delta_{1}\right) f(x-y) .
$$

By (8) and (9), we have

$$
\begin{gathered}
f(x-y)-f(A x-A y) \\
\geq f(x-y)-\psi\left(\delta_{1}\right) f(x-y) \geq\left(1-\psi\left(\delta_{1}\right)\right) \delta_{1} .
\end{gathered}
$$

Property (P4) and (7) imply that

$$
\|x-A x\| \leq \delta_{2} \text { and }\|y-A y\| \leq \delta_{2} .
$$

In view of (6) and (11), we have

$$
|f(x-y)-f(A x-A y)| \leq\left(1-\psi\left(\delta_{1}\right)\right) \delta_{1} / 2 .
$$

This inequality, however, contradicts (10). The contradiction we have reached proves that

$$
\|x-y\| \leq \epsilon
$$

and that property (P3) holds.

Let $x \in K$. In view of (4),

$$
\lim _{n \rightarrow \infty} f\left(A^{n} x-A^{n+1} x\right)=0 .
$$

When combined with property (P3), this implies that $\left\{A^{n} x\right\}_{n=1}^{\infty}$ is a Cauchy sequence. Therefore,

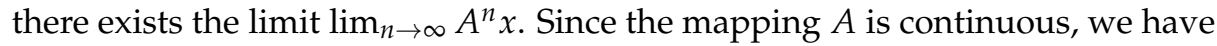

$$
A\left(\lim _{n \rightarrow \infty} A^{n} x\right)=\lim _{n \rightarrow \infty} A^{n} x
$$

and $\lim _{n \rightarrow \infty} A^{n} x$ is a fixed point of the mapping $A$. Property (P3) now implies the uniqueness of the fixed point of $A$. Therefore there exists a point $x_{A} \in K$ such that

$$
A x_{A}=x_{A}
$$

and for each $x \in K$,

$$
\lim _{n \rightarrow \infty} A^{n} x=x_{A} .
$$

We claim that

$$
A^{n} x \rightarrow x_{A} \text { as } n \rightarrow \infty,
$$

uniformly on all bounded subsets of $K$.

Let $M, \epsilon>0$. By (1) and (12), for all $x \in K$,

$$
f\left(A x-x_{A}\right) \leq \psi\left(f\left(x-x_{A}\right)\right) f\left(x-x_{A}\right) .
$$

By property (P1), there is $\epsilon_{1} \in(0, \epsilon)$ for which

$$
\|x-y\| \leq \epsilon \text { for all } x, y \in K \text { satisfying } f(x-y) \leq \epsilon_{1} .
$$


In view of (P2), there is $c_{0}>0$ for which

$$
f\left(z-x_{A}\right) \leq c_{0} \text { for all } x \in K \text { satisfying }\left\|z-x_{A}\right\| \leq M \text {. }
$$

Choose an integer

$$
n(M, \epsilon)>1+\left(c_{0}+M\right) \epsilon_{1}^{-1}\left(1-\psi\left(\epsilon_{1}\right)\right)^{-1}
$$

and let a point $x \in K$ satisfy

$$
\left\|x-x_{A}\right\| \leq M .
$$

We claim that for all integers $n \geq n(M, \epsilon)$, we have

$$
\left\|x_{A}-A^{n} x\right\| \leq \epsilon .
$$

In view of (15), it suffices to show that for all integers $n \geq n(M, \epsilon)$,

$$
f\left(A^{n} x-x_{A}\right) \leq \epsilon_{1} .
$$

By (14), in order to establish this inequality, it is enough to prove that there is an integer

$$
m \in[0, n(M, \epsilon)]
$$

for which

$$
f\left(A^{m} x-x_{A}\right) \leq \epsilon_{1} .
$$

Suppose, to the contrary, that this is not true. Then for each $i \in\{0, \ldots, n(M, \epsilon)\}$, we have

$$
f\left(A^{i} x-x_{A}\right)>\epsilon_{1} .
$$

Since the function $\psi$ is decreasing, it follows from (14) and (19) that for each $i \in\{0, \ldots, n(M, \epsilon)\}$, we have

$$
\begin{gathered}
f\left(A^{i+1} x-x_{A}\right) \leq \psi\left(f\left(A^{i} x-x_{A}\right)\right) f\left(A^{i} x-x_{A}\right) \\
\leq \psi\left(\epsilon_{1}\right) f\left(A^{i} x-x_{A}\right)
\end{gathered}
$$

and

$$
\begin{gathered}
f\left(A^{i} x-x_{A}\right)-f\left(A^{i+1} x-x_{A}\right) \\
\geq\left(1-\psi\left(\epsilon_{1}\right)\right) f\left(A^{i} x-x_{A}\right) \geq \epsilon_{1}\left(1-\psi\left(\epsilon_{1}\right)\right) .
\end{gathered}
$$

By (16), (18), and (20),

$$
\begin{gathered}
c_{0} \geq f\left(x-x_{A}\right) \geq f\left(x-x_{A}\right)-f\left(A^{n(M, \epsilon)} x-x_{A}\right) \\
=\sum_{i=0}^{n(M, \epsilon)-1}\left(f\left(A^{i} x-x_{A}\right)-f\left(A^{i+1)} x-x_{A}\right)\right) \\
\geq \epsilon_{1}\left(1-\psi\left(\epsilon_{1}\right)\right) n(M, \epsilon)
\end{gathered}
$$

and

$$
n(M, \epsilon) \leq c_{0} \epsilon_{1}^{-1}\left(1-\psi\left(\epsilon_{1}\right)\right)^{-1} .
$$

This, however, contradicts (17). The contradiction we have reached completes the proof of Theorem 1. 
Author Contributions: These authors contributed equally to all aspects of this work. All authors have read and agreed to the published version of the manuscript.

Funding: The first author was supported by the Israel Science Foundation (Grant No. 820/17), the Technion General Research Fund and by the Fund for the Promotion of Research at the Technion.

Acknowledgments: Both authors thank the three referees for their helpful comments and useful suggestions.

Conflicts of Interest: The authors declare no conflict of interest.

\section{References}

1. Betiuk-Pilarska, A.; Domínguez Benavides, T. Fixed points for nonexpansive mappings and generalized nonexpansive mappings on Banach lattices. Pure Appl. Funct. Anal. 2016, 1, 343-359.

2. Butnariu, D.; Reich, S.; Zaslavski, A.J. Convergence to fixed points of inexact orbits of Bregman-monotone and of nonexpansive operators in Banach spaces. In Proceedings of the Seventh International Conference on Fixed Point Theory and its Applications, Guanajuato, Mexico, 17-23 July 2005; pp. 11-32.

3. De Blasi, F.S.; Myjak, J.; Reich, S.; Zaslavski, A.J. Generic existence and approximation of fixed points for nonexpansive set-valued maps. Set-Valued Var. Anal. 2009, 17, 97-112. [CrossRef]

4. Goebel, K.; Kirk, W.A. Topics in Metric Fixed Point Theory; Cambridge University Press: Cambridge, UK, 1990.

5. Goebel, K.; Reich, S. Uniform Convexity, Hyperbolic Geometry, and Nonexpansive Mappings; Marcel Dekker: New York, NY, USA; Basel, Switzerland, 1984.

6. Jachymski, J. Extensions of the Dugundji-Granas and Nadler's theorems on the continuity of fixed points. Pure Appl. Funct. Anal. 2017, 2, 657-666.

7. Kirk, W.A. Contraction mappings and extensions. In Handbook of Metric Fixed Point Theory; Kluwer: Dordrecht, The Netherlands, 2001; pp. 1-34.

8. Kubota, R.; Takahashi, W.; Takeuchi, Y. Extensions of Browder's demiclosedness principle and Reich's lemma and their applications. Pure Appl. Funct. Anal. 2016, 1, 63-84.

9. Pustylnyk, E.; Reich, S.; Zaslavski, A.J. Convergence to compact sets of inexact orbits of nonexpansive mappings in Banach and metric spaces. Fixed Point Theory Appl. 2008, 2008, 528614. [CrossRef]

10. Reich, S.; Zaslavski, A.J. Generic well-posedness of fixed point problems. Vietnam J. Math. 2018, 46, 5-13. [CrossRef]

11. Reich, S.; Zaslavski, A.J. Generic aspects of metric fixed point theory. In Handbook of Metric Fixed Point Theory; Kluwer: Dordrecht, The Netherlands, 2001; pp. 557-575.

12. Reich, S.; Zaslavski, A.J. Convergence to attractors under perturbations. Commun. Math. Anal. 2011, 10, 57-63.

13. Reich, S.; Zaslavski, A.J. Genericity in Nonlinear Analysis; Developments in Mathematics; Springer: New York, NY, USA, 2014; Volume 34.

14. Zaslavski, A.J. Approximate Solutions of Common Fixed Point Problems; Springer Optimization and Its Applications; Springer: Cham, Switzerland, 2016.

15. Zaslavski, A.J. Algorithms for Solving Common Fixed Point Problems; Springer Optimization and Its Applications; Springer: Cham, Switzerland, 2018.

16. Banach, S. Sur les opérations dans les ensembles abstraits et leur application aux équations intégrales. Fund. Math. 1922, 3, 133-181. [CrossRef]

17. Censor, Y.; Zaknoon, M. Algorithms and convergence results of projection methods for inconsistent feasibility problems: A review. Pure Appl. Funct. Anal. 2018, 3, 565-586.

18. Gibali, A. A new split inverse problem and an application to least intensity feasible solutions. Pure Appl. Funct. Anal. 2017, 2, 243-258.

19. Gibali, A.; Reich, S.; Zalas, R. outer approximation methods for solving variational inequalities in Hilbert space. Optimization 2017, 66, 417-437. [CrossRef]

20. Takahashi, W. The split common fixed point problem and the shrinking projection method for new nonlinear mappings in two Banach spaces. Pure Appl. Funct. Anal. 2017, 2, 685-699.

21. Takahashi, W. A general iterative method for split common fixed point problems in Hilbert spaces and applications. Pure Appl. Funct. Anal. 2018, 3, 349-369.

22. Reich, S.; Zaslavski, A.J. Contractivity and genericity results for a class of nonlinear mappings. J. Nonlinear Convex Anal. 2015, 16, 1113-1122. 
23. Gabour, M.; Reich, S.; Zaslavski, A.J. A generic fixed point theorem. Indian J. Math. 2014, 56, 25-32.

24. De Blasi, F.S.; Myjak, J. Sur la convergence des approximations successives pour les contractions non linéaires dans un espace de Banach. C. R. Acad. Sci. Paris 1976, 283, 185-187.

25. Reich, S.; Zaslavski, A.J. Well-posedness of generalized best approximation problems. Nonlinear Funct. Anal. Appl. 2002, 7, 115-128.

26. Reich, S.; Zaslavski, A.J. Porous sets and generalized best approximation problems. Nonlinear Anal. Forum 2004, 9, 135-152.

27. Rakotch, E. A note on contractive mappings. Proc. Am. Math. Soc. 1962, 13, 459-465. [CrossRef]

(c) 2020 by the authors. Licensee MDPI, Basel, Switzerland. This article is an open access article distributed under the terms and conditions of the Creative Commons Attribution (CC BY) license (http:/ / creativecommons.org/licenses/by/4.0/). 